\title{
Analysis of Epidemiological Changes and Elimination Effects for Malaria in North China
}

\author{
Ruiling Guo \\ Handan Municipal Centre for Disease Control and Prevention \\ Jing Zhuang \\ Handan Municipal Centre for Disease Control and Prevention \\ Xianfeng Wang \\ Hebei University of Engineering \\ Jinsheng Liu \\ Hebei University of Engineering \\ Wangdong Zhu \\ Hebei University of Engineering \\ Shuang Liang \\ Hebei University of Engineering \\ Zengjun Jin ( $\boldsymbol{\nabla}$ jinzengjun@hebeu.edu.cn ) \\ Hebei University of Engineering
}

\section{Research Article}

Keywords: Malaria, Imported malaria, Surveillance, Evaluation, Elimination

Posted Date: August 4th, 2021

DOl: https://doi.org/10.21203/rs.3.rs-762882/v1

License: (c) (1) This work is licensed under a Creative Commons Attribution 4.0 International License.

Read Full License 
2 Analysis of epidemiological changes and elimination effects for malaria in North

3 China

4

5 Ruiling Guo ${ }^{1}$, Jing Zhuang ${ }^{1}$, Xianfeng Wang $^{2}$,3, Jinsheng Liu ${ }^{2,3}$, Wangdong Zhu ${ }^{2,3}$,

6 Shuang Liang $2,3, *$, Zengjun $\mathrm{Jin}^{2,3, *}$

7

$8{ }^{1}$ Handan Municipal Centre for Disease Control and Prevention, Handan 056008, 9 Hebei, China. ${ }^{2}$ School of Medicine, Hebei University of Engineering, Handan 056038 , 10 Hebei, China. 3 Handan Key Laboratory of Integrated Medical and Industrial 11 Application in Basic Medicine.

12

13

14

15

16

17

18

19

20

21

22

*Correspondence: yxykyb@ hebeu.edu.cn, jinzengjun@hebeu.edu.cn 


\section{Abstract}

Backgroud: The elimination of malaria requires high-quality surveillance data in order to detect and respond quickly to individual cases. In the 1960s and 1970s, Handan city in North China experienced widespread malaria outbreaks, which was in line with the provincial and national epidemic patterns.

Methods: Case-level data for the period 2011-2018 were extracted from China's National Infectious Diseases Information system and the rest cases were recorded municipal surveillance system from 1956-2010.

Results: The incidence, accuracy and timeliness of case diagnosis, reporting and investigation in malaria were evaluated at elimination stage (2011-2018) in Handan city, China. From 2011 to 2018, 81 malaria cases were reported in Handan city, all of which were imported from abroad. The annual average incidence decreased to $0.11 / 100000$ in the elimination stage, while all malaria cases were male. Since the initiation of the National Malaria Elimination Programme in 2010, malaria cases were consistent with the increase in overseas export channels and personnel of labor services.

Conclusions: The case-based malaria surveillance system in North China worked well at the malaria elimination stage. This ensured that malaria cases could be diagnosed, reported and timely investigated at local level.

Keywords: Malaria, Imported malaria, Surveillance, Evaluation, Elimination 


\section{Backgroud}

Malaria is considered one of the most significant health problems for humans with a substantial disease burden in tropical areas. Malaria results from a vector borne plasmodial infection with single-celled parasites belonging to the Plasmodium genus and transmitted via the bites of the female Anopheles mosquito[1]. In 2017, there were approximately 219 million cases of malaria responsible for about 435000 deaths, the majority on the African. Outstanding progress has been made in malaria control over the past decade[2-4]. More and more countries are progressing to elimination. As of December 2017, of the 106 countries with sustained transmission of malaria in 2000, 19 countries attained zero indigenous cases for 3 years or more; 16 of these countries that eliminated malaria between 2007 and 2017. To achieve the goal of eliminating malaria, a sustainable and well-functioning malaria monitoring system is considered as a key measure[5]. World Health Organization (WHO) launched a new initiative on global malaria programme 3T, Test, Treatment and Track in 2012, which depends on the provision of timely and accurate monitoring data to monitor performance and identify threats to malaria control and elimination[6].

In the past, especially in the last 30-40 years after the founding of the People's Republic of China, China has suffered from severe malaria epidemics[7]. From 1949 to 2020 , the transmission of the malaria can be primarily devided into four phases: transmission; outbreak and pandemic transmission; decline with sporadic distribution; and the elimination phase[8]. A national malaria elimination programme (NMEP) was launched in China in 2010, which surports to achieve zero indigenous case of malaria 
within the 2020 timeline[9]. The elimination phase is different from the control phase, and needs to monitor and respond to each malaria infection, and to eventually stop local malaria transmission. China has developed a case-based malaria surveillance system to gather information needed for diagnosis and investigation, and to promote a rapid response to individual cases[10, 11]. In order to eliminate malaria, the strengths and limitations of the program must be understood by quantitatively evaluating the performance and efficiency of NMEP[12, 13].

According to the report from WHO in 2018, there were 20 million fewer cases in 2017 than in 2010 globally. At the same time, increasing labour service and travel to malaria-endemic areas in recent decades has resulted in a steady increase in the number of imported cases in non-endemic countries[14]. Malaria poses a serious health hazard to travelers to local areas. Imported malaria is an infection acquired abroad and brought into the regional territory[15]. Cases imported-malaria into non-endemic countries tend to lead to delayed diagnosis, expensive treatment and, sometimes, secondary local transmission[16]. During 2017-2019, China had achieved interruption of indigenous malaria transmission. Still, malaria is the imported disease with the highest number of notifications in China, between 2500 and 10000 malaria cases are imported into China from 2002 to 2017[9].

Given an example, Handan city (2018 population 9.52 million), a typical northern city with a long history in North China. Based on literature records and data analysis, the last local malaria case in Handan was occurred before 2005. In the last decades, notified malaria infections have been entirely imported. There was no documented 
cases by autochthonous transmission, and it mainly related to the imrovement of public health care and a strong sense of personal health. Although there is a partial distribution of the potential vector of this species, it is considered that the current risk of introduced malaria is very low[17]. In order to improve knowledge about malaria-imported cases characteristics, the epidemiological and clinical characteristics of patients diagnosed with malaria in North China were assessed.

In this study, an epidemiological investigation of every case of imported malaria in North China was conducted. We have a systematic analysis of the key components of malaria surveillance, including trends in malaria prevalence, the origin of imported malaria, the Plasmodium species, and prevention effects of malaria.

\section{Methods}

\section{Study site}

Handan city is located in the southern end of Hebei Province and in the northtern part of China, laying between latitudes $36^{\circ} 20^{\prime}$ and $36^{\circ} 40^{\circ} \mathrm{N}$, and longitude $114^{\circ} 03^{\prime}$ and $114^{\circ} 40^{\circ} \mathrm{E}$. The total area is 12047 square kilometers, of which the mountainous area is 4460 square kilometers, the plain area is 7587 square kilometers. It has apopulation of $>9.5$ million and includes 18 counties. Handan belongs to the southeast monsoon climate region, which is a continental monsoon climate transiting from the semi-humid zone of warm zone to the semi-arid zone. It has abundant sunshine, the same period of rain and heat, the same period of dry and cold, and the four seasons are alternating obviously. The main cash crops wheat, maize, cotton, millet, rice and soybean. This variety in climate, environment, and ecology makes the area favourable 
for mosquitoes.

\section{Study design}

The data were obtained from the national malaria surveillance system, including two systems: National notifiable infectious disease reporting information system and Malaria enhanced surveillance information system, and compilation of information on the elimination of malaria, (i.e., epidemic situation reports, individual case information reports and assessment reports of malaria elimination).

\section{Fever patient blood smear microscopy}

Since 2010, microscopic examinations on "triple fever" patients (clinically diagnosed as having malaria, suspected malaria or unexplained fever) have been carried out each year. And blood tests have been conducted for malaria parasites.

\section{Mosquito collection and species identification}

The lamp trap method was used. Methods According to the characteristics of mosquito breeding and distribution, four representative areas with different habitats were selected in residential areas, parks, hospitals, rural houses and cattle sheds in three urban districts and rural suburbs of Handan. The survey will be conducted twice a month from May to October every year.In the above time, one mosquito lamp was used in each place. The monitoring began 30 minutes after sunset, and the captured adult mosquitoes were collected continuously for 12 hours. The captured adult mosquitoes were brought back to the laboratory and smoked with ether for classification and identification.

Light-traps without bait and landing count method were used to capture mosquitoes. After being transferred to the laboratory, the mosquitoes were morphologically divided into Anopheles, Culex, Aedes, and other subfamilies or genera. The species of 
Anopheles were further classified by morphology[18].

\section{Data analysis}

All data analysis was conducted by the Statistical Package for Social Sciences software (version 22.0; SPSS Inc., IL, USA).

\section{Ethics approval and consent to participate}

The experimental research reported in the current study was performed with the approval of the Ethics Committee of the Handan Municipal Centre for Disease Control and Prevention and the Ethics Committee of Hebei University of Engineering. Human research was performed in compliance with the Declaration of Helsinki and its later amendments. All participants provided their written informed consent to participate in this research.

\section{Results}

\section{Descriptive analysis of the malaria cases}

\section{Occurrence of cases}

In the 1960s and 1970s, Handan city experienced widespread malaria outbreaks, which was in line with the provincial and national epidemic patterns. From 1956 to 2010, a total of 1029532 malaria cases were reported in Handan city, accounting for more than $11 \%$ of the total malaria cases in North China during the same period. The prevalence of malaria was mainly from the 1950 s to the $1980 \mathrm{~s}$, and the average incidence of malaria in each decade was 119.9/100 000, 1079.8/100 000, 980.5/100 000 and 7.7/100 000, respectively. During this period, there were three epidemic peaks in Handan city, respectively in 1964, 1970 and 1973, and the reported incidence was as high as 3135.5/100 000, 2281.9/100 000 and 1833.0/100 000, respectively. After 1973, the annual incidence of malaria declined rapidly. By 1985, the annual 
(Figure 1A).

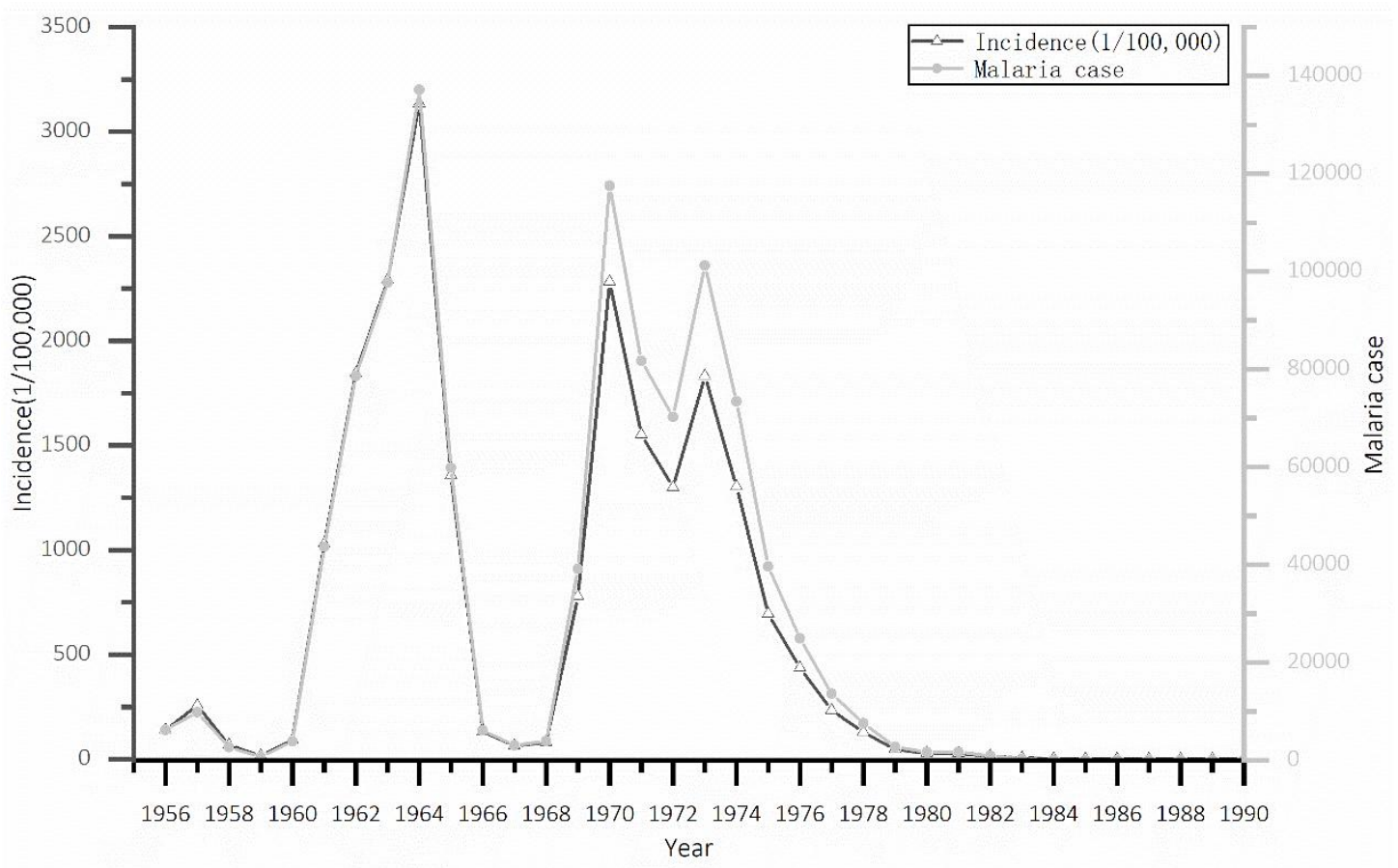

162 Figure 1A Malaria incidence and cases reported in Handan, Hebei Province from 1631956 to 1990.

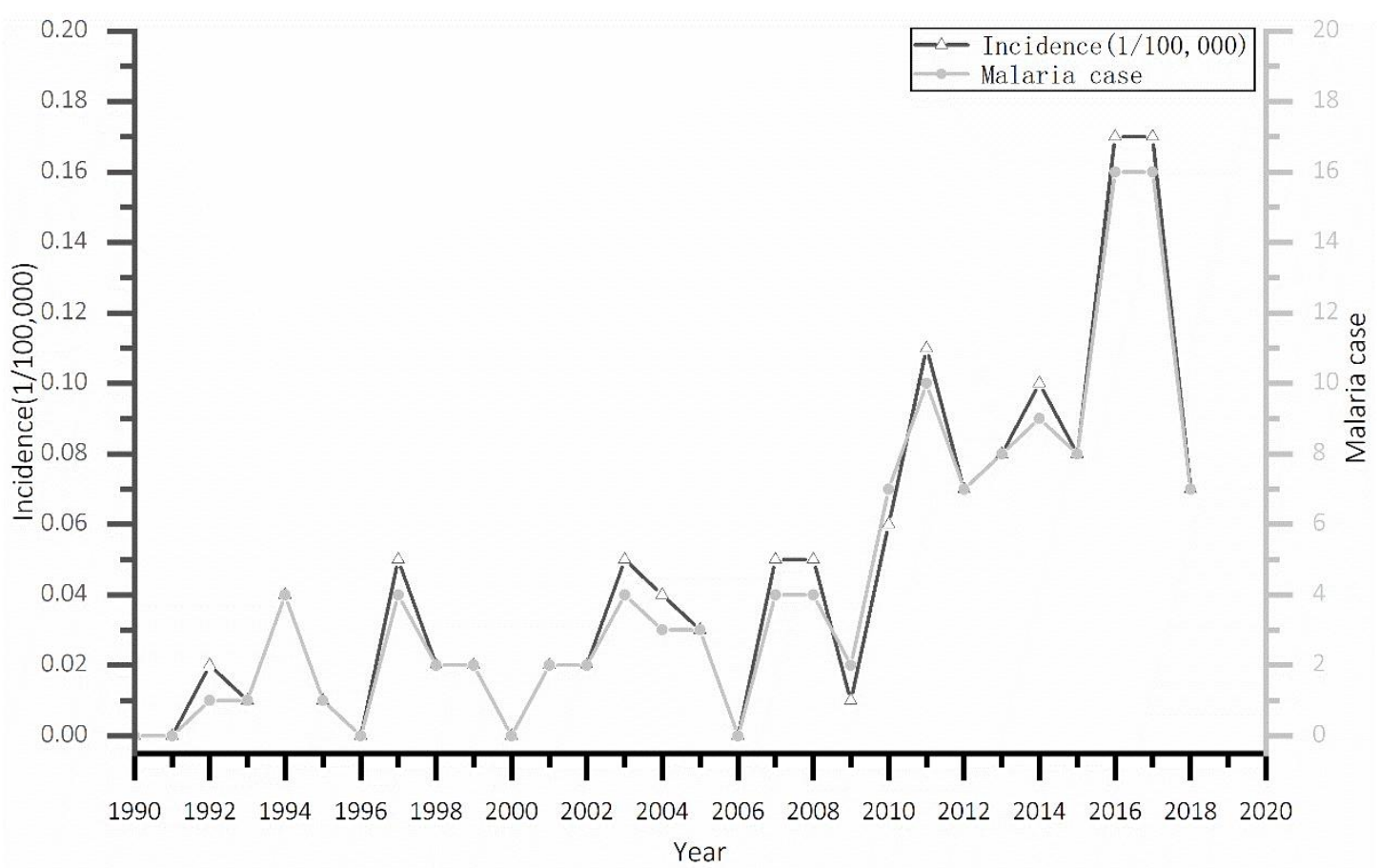

Figure 1B Malaria incidence and cases reported in Handan, Hebei Province from 
1991 to 2018.

During 1989-2010, 47 cases of malaria were reported in the early malaria elimination phase. The highest incidence rate was $2010(0.06 / 100000)$, and no malaria cases were reported in 1990, 1991, 1996, 2000 and 2006. In the eradication phase between 2011 and 2018, a total of 81 imported cases of malaria were reported (Figure 1B). After 2009, there was an increase in fluctuation of malaria cases imported from other countries.

\section{Determinaton/Judgement of the final indigenous case}

All 81 malaria cases reported in Handan City from 2011 to 2018 had detailed epidemiological records, and all of them had a detailed history of living in high malaria areas abroad, which could rule out the possibility of local infection.

From 2005 to 2010, there were 17 cases of malaria in Handan city, 5 cases of plasmodium falciparum malaria, 2 cases of plasmodium vivax malaria, 1 case of mixed infection of $P$. vivax and $P$. falciparum malaria, and 9 cases of unclassified malaria. Since our city was historically an endemic area of $P$. vivax and no $P$. falciparum malaria vector existed, we determined that the P. falciparum cases were imported cases. A retrospective investigation was conducted on the outward travel history of 2 cases of $P$. vivax malaria and 9 cases of unclassified malaria. The survey confirmed they all had experience of working in malaria-infected areas in south China or abroad. According to the above investigation results, it can be concluded that the last indigenous case in Handan city occurred before 2005 .

\section{Plasmodium species composition}


188 From 1956 to 2018, four species of plasmodium (P. vivax, P. falciparum, P. ovale and 189 P. malariae) were identified in 1029613 malaria cases. Prior to 1988 , all cases of 190 malaria were caused by $P$. vivax infection and included 1029485 cases. From 1989 to 1912010 , a total of 47 cases of malaria have been reported. And one P. falciparum case 192 and one unclassified Plasmodium case has been reported in 2007. From 2011 to 2018, 19355 cases of $P$. falciparum accounted for $67.9 \%$ of the total malaria infections. In 194 addition, three cases of $P$. ovale infection and one case of $P$. malariae infection was 195 identified during this period. Furthermore, there were nine unclassified plasmodium 196 infections (Table 1).

197 Table 1 Plasmodium species in Handan city, 1956-2018

\begin{tabular}{cccccccc}
\hline Year & P. vivax & $\boldsymbol{P}$. falciparum & $\boldsymbol{P}$ & $\boldsymbol{P}$. & & & \\
& & & ovale & malariae & & & \\
\hline $1956-1970$ & 609778 & 0 & 0 & 0 & 0 & 0 & 609778 \\
$1971-1988$ & 419707 & 0 & 0 & 0 & 0 & 0 & 419707 \\
$1989-2006$ & 30 & 0 & 0 & 0 & 0 & 0 & 30 \\
$2007-2018$ & 17 & 61 & 3 & 1 & 7 & 9 & 98 \\
Total & 1029532 & 61 & 3 & 1 & 7 & 9 & 1029613 \\
\hline
\end{tabular}

201 Among the 81 cases, there were 14 cases of $P$. vivax, 55 cases of $P$. falciparum, 3

202 cases of $P$. ovale, 7 cases of $P$. falciparum and $P$. ovale mixed infection, 1 case of $P$. 
malariae, and 1 case of unclassified during 2011-2018 (Figure 2). Of these, 37 cases were reported locally and 44 were reported from other cities. The demographic features of imported cases in 2011-2018 was shown in Table 2. All of the imported malaria cases were male. The 21-50 age group had the most malaria cases, accounting for $91.4 \%$ of the total 81 cases. The remaining age groups of 20 years or less and 51 years or more included only a small number of cases, 1 and 6 , respectively. The proportion of farmers, oversea laborers and workers was the highest, accounting for $43.2 \%(35 / 81), 24.7 \%(20 / 81)$ and $19.8 \%(16 / 81)$ of the total occupation distribution, respectively.

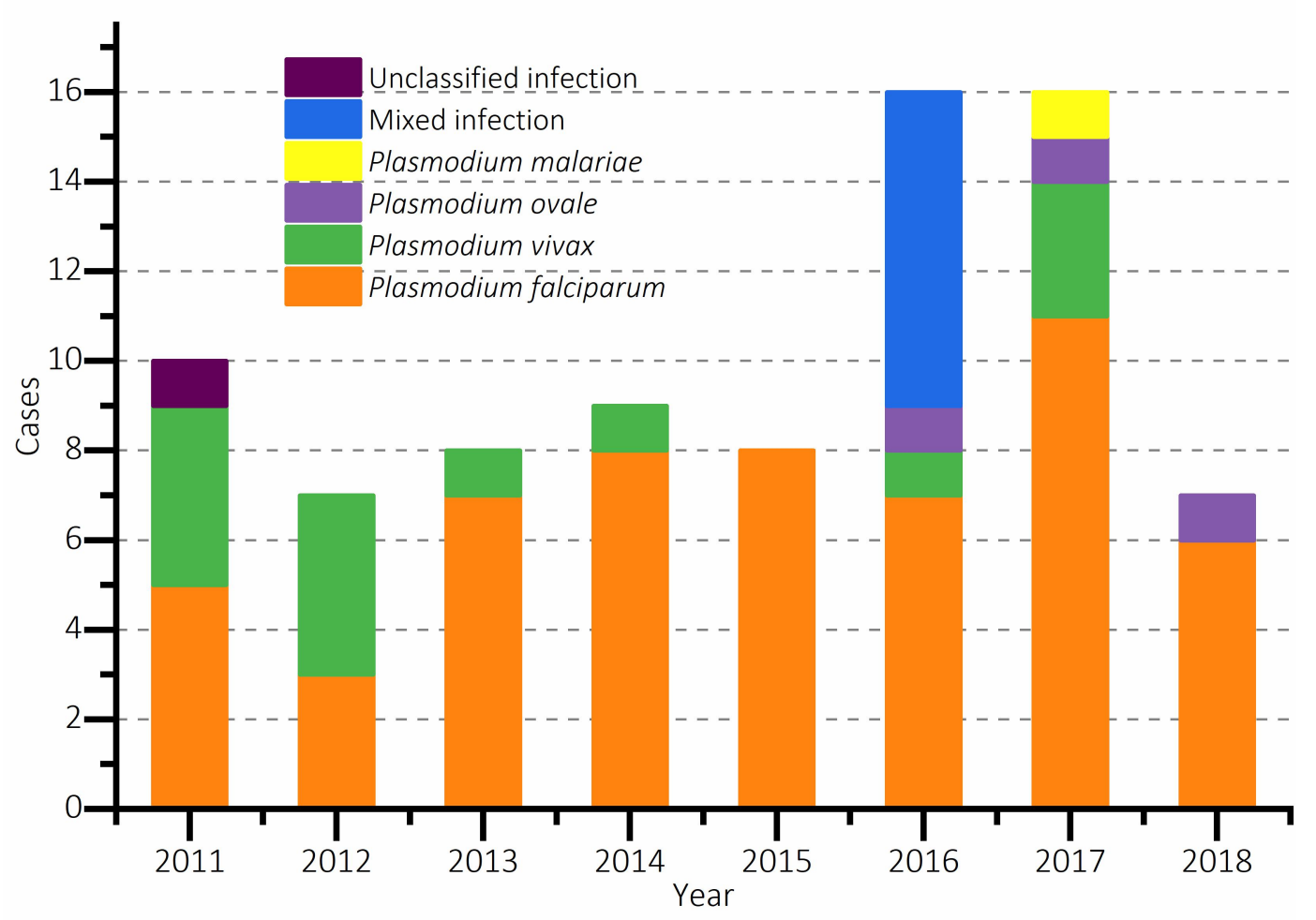

Figure 2 Species type distribution of imported malaria in Handan city from 2011 to 2018.

Table 2 Demographic characteristic of malaria cases in Handan city between 2011 and 2018. 


\begin{tabular}{cccccccccc}
\hline Variables & $\mathbf{2 0 1 1}$ & $\mathbf{2 0 1 2}$ & $\mathbf{2 0 1 3}$ & $\mathbf{2 0 1 4}$ & $\mathbf{2 0 1 5}$ & $\mathbf{2 0 1 6}$ & $\mathbf{2 0 1 7}$ & $\mathbf{2 0 1 8}$ & Total \\
\hline Male & 10 & 7 & 8 & 9 & 8 & 16 & 16 & 7 & 81 \\
$\leqslant 20$ & 0 & 0 & 1 & 0 & 0 & 0 & 0 & 0 & 1 \\
$21-50$ & 10 & 7 & 7 & 9 & 7 & 15 & 14 & 5 & 74 \\
$\geqslant 51$ & 0 & 0 & 0 & 0 & 1 & 1 & 2 & 2 & 6 \\
Farmers & 1 & 1 & 5 & 5 & 3 & 11 & 9 & 0 & 35 \\
Oversea laborers & 8 & 3 & 0 & 2 & 3 & 3 & 1 & 0 & 20 \\
Workers & 1 & 0 & 1 & 1 & 1 & 1 & 4 & 7 & 16 \\
Houseworker & 0 & 1 & 2 & 0 & 1 & 0 & & 0 & 4 \\
Businessmen & 0 & 1 & 0 & 1 & 0 & 1 & & 0 & 3 \\
Others & 0 & 1 & 0 & 0 & 0 & 0 & 2 & 0 & 3 \\
\hline
\end{tabular}

*Others include cadres, Individual worker, for example.

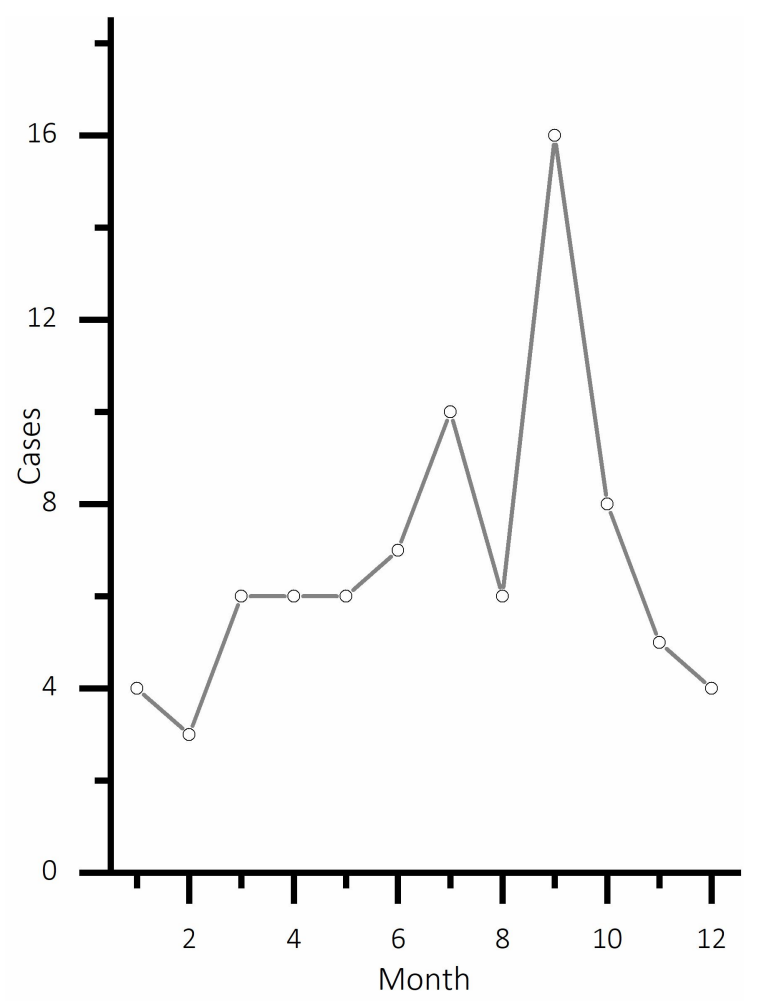

219 Figure 3 Monthly distribution of imported malaria in Handan city during the period of 
2011-2018. every month during 2011-2018. There were 10 cases in July and 16 cases in September, and 3-8 cases in other months, which was consistent with the time of overseas return of the cases (Figure 3). In addition, the 8-year cases analysis displayed that most of these cases were recorded in the counties of Chengan, Linzhang, Weixian and the main city zone of Handan, which reported 41, 8,5 and 8 cases of imported malaria, respectively, which accounted for $76.5 \%$ of the total number of cases. All the imported cases had work experience abroad before their onset. Among them, 22 cases were from Nigeria and 20 cases from Congo, accounting for 51.8\%. Angola, Pakistan and Myanmar followed with 5, 6 and 5 cases, respectively. The results of epidemiological investigation showed that all the cases were imported from abroad.

\section{Case investigations}

All 37 cases were investigated by malaria control personnel of the disease control institutions in their jurisdiction, and the samples of cases were rechecked by county, city and provincial CDC. After a laboratory confirmed malaria case, the municipal CDC provides the patient with antimalarial drugs. The clinician is responsible for the treatment of the patient, and the malaria control personnel in the district are responsible for the follow-up of the treatment progress and results of the case. In view of the historical prevalence of $P$. vivax in our city, and the occasional capture of Anopheles sinensis (An. sinensis) in mosquito density monitoring in recent years, we determined the living places of these $9 P$. vivax cases to be the epidemic sites with the 
242 possibility of transmission, and carried out key treatment according to the disposal

243 requirements. An. sinensis and other malaria mosquitoes were not found by vector

244 investigation, and no suspicious cases were found in active screening of fever cases.

245 The standard disposal rate of the epidemic site was $100 \%$, and no imported secondary

246 cases of locally infected malaria were detected.

$247 \quad$ Fever patient blood tests

248 According to province program requirements, the number of blood fever patients

249 between 2011 and 2018 was higher than blood tests required. Except for 2011, the

250 completion rate of blood tests was more than $100 \%$, and the proportion of blood tests

251 in the transmission season reached 50\%. Since 2010, Handan has been actively

252 managing patients who are currently ill. 81 malaria cases were treated, including 37

253 locally reported imported cases, and all cases were in good condition after treatment

254 (Table 3).

255 Table 3 The number of blood tests and patient treated in Handan city, 2011-2018

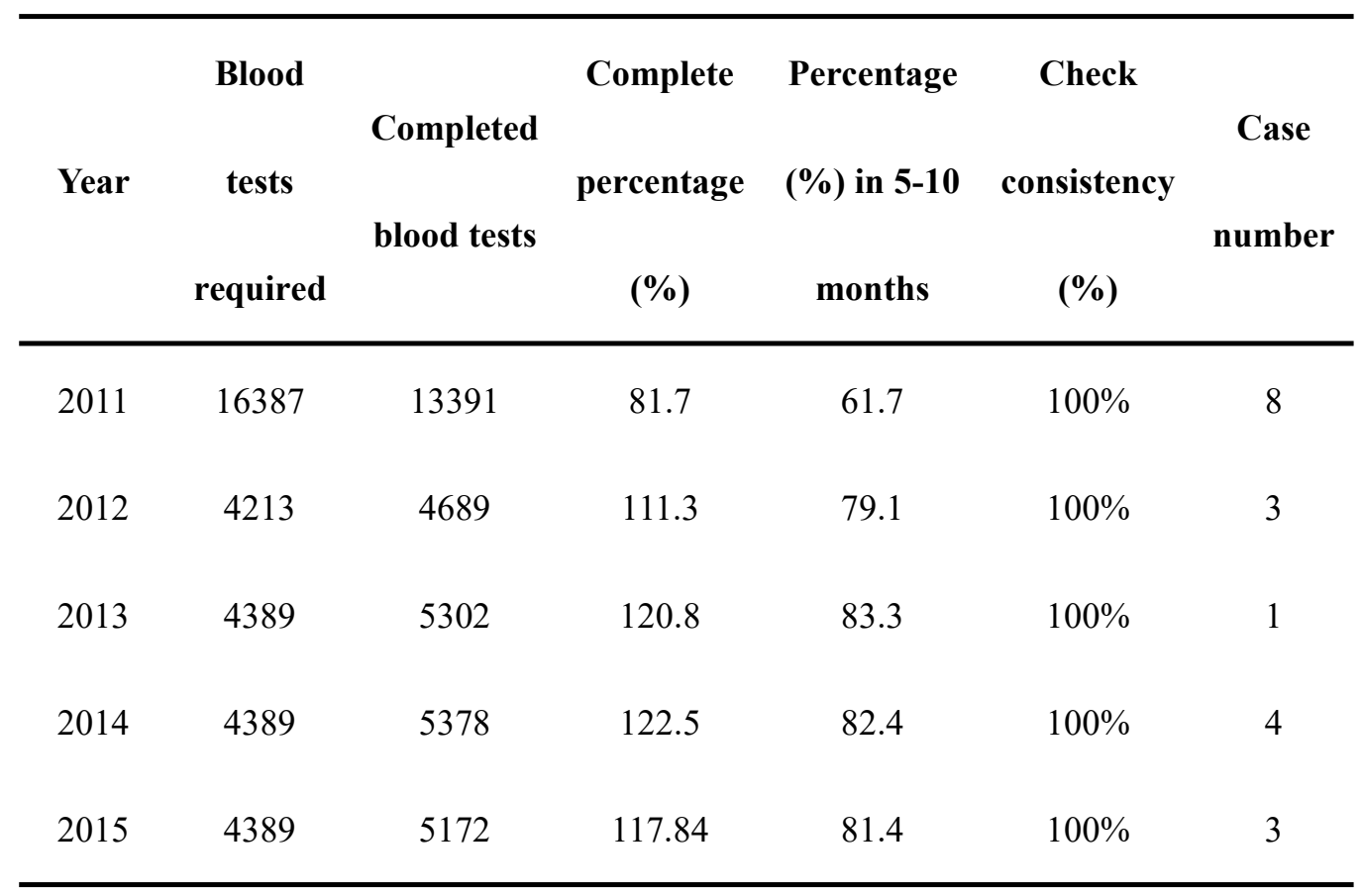




\begin{tabular}{ccccccc}
\hline 2016 & 950 & 1073 & 113.0 & 50.0 & $100 \%$ & 7 \\
2017 & 900 & 1046 & 116.2 & 53.4 & $100 \%$ & 8 \\
2018 & 900 & 1214 & 134.9 & 62.3 & $100 \%$ & 3 \\
Total & 36517 & 37265 & & & & 37 \\
\hline
\end{tabular}

\section{Vector investigations}

258 During the malaria epidemic in the 20th century, all the malaria cases in Hebei 259 province were $p$. vivax, and An. sinensis was the only malaria vector. Traceable vector 260 monitoring data in Handan city began in the 1950s. There were 18 species of adult 261 mosquitoes from3 genera and 18 species of larvae. Except An. sinensis, no other 262 species of malaria vector were found. The main breeding places of An. sinensis are 263 concentrated in rice fields and its irrigation systems. According to the number of $A n$. 264 sinensis specimens and the date and place of collection, An. sinensis is a common 265 mosquito species in Handan City. Before the year of 1993, rice cultivation area in the 266 Fu River and Qing Zhang River basin was large. With the rapid social development,

267 rice cultivation has been greatly reduced since 2003 . Now, there are almost no paddy 268 fields, but mainly wheat and corn. 


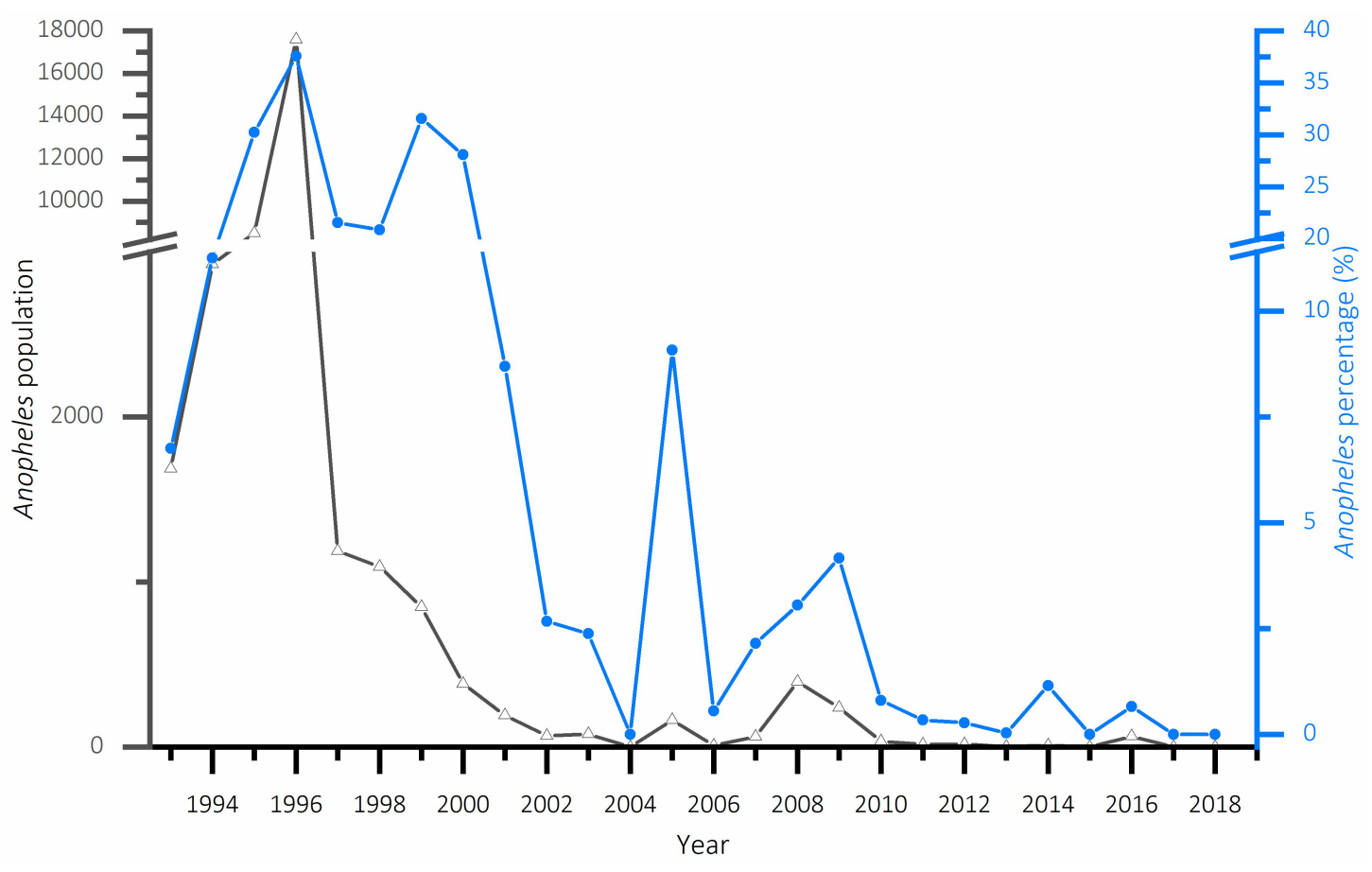

Figure 4 Anopheles number and Anopheles percentage accounting for the captured mosquitoes in Handan, Hebei Province in the 1993-2018 period.

272 Since 1993, mosquito vector monitoring has been carried out continuously in the main urban area of Handan, the annual mosquito density fluctuates greatly, but the overall trend is decreasing, and it is related to the annual precipitation (Figure 4). In 1996, during the flood disaster in Handan City, the highest number of An. sinensis mosquitoes were captured, with a density of 251.3 mosquitoes per night lamp, accounting for $37.6 \%$ of the captured mosquitoes. After that, both the density and proportion of Anopheles mosquitoes showed a decreasing trend. According to the monitoring results from 2003 to 2015, the composition ratio and capture number of An. sinensis have decreased significantly since 2010 . The density of mosquitoes, the

281 density of Anopheles and the proportion of Anopheles increased slightly during the 282 floods in Handan City in the summer of 2016. Subsequently, An. sinensis was not caught in 2017 and 2018. 


\section{Discussion}

Handan used to be an area of high prevalence of $P$. vivax, with An. sinensis as the single vector. The rivers in Handan are Ming River in the north, Fuyang River in the middle and Zhang River in the south. At present, Fuyang River has water all the year round, and Ming River and Zhang River are in a dry period. However, tributaries with reeds, irrigation ditches, ponds and drains around villages are the best breeding grounds for An. sinensis. And people who live nearby are more likely to get bitten by mosquitoes during peak biting times, which can lead to malaria. In Handan City, corn and wheat are the main crops. In the 1970s, Fuyang River basin began to introduce rice planting and formed a scale of water field; with the reduction of surface water resources and ecological environment control, water field has been basically disappeared by the end of the 1990s. This is also a good fit with the malaria epidemic trend in our city.

During the epidemic stage (1956-1985), the incidence of malaria in Handan was obviously seasonal, with high incidence from May to September. With the increase of national efforts to prevent and control infectious diseases and the improvement of the environment, the incidence of malaria dropped to sporadic levels in the late 1980s. The incidence was non-seasonal, and cases were reported even in winter and spring when mosquitoes were not active. From 1989 to 2004, a total of 27 malaria cases were reported. Due to the lack of relevant epidemiological data, it is impossible to verify whether the malaria cases reported during this period were locally infected.

A total of 20 malaria cases were reported in 2005-2010. According to the case 
information, there was a clear history of living in areas with high malaria in foreign countries and they were imported cases. From 2011 to 2018, 81 cases were reported, all of which had detailed epidemiological investigation information and were imported from abroad. The number of imported malaria cases increased during this period, which was consistent with the increase of export channels and personnel of labor services abroad. In this period, malaria elimination efforts will focus on the management of malaria patients, the maintenance of blood testing capacity for malaria parasites in patients with fever, and the screening and investigation of people traveling to and from areas with high malaria levels.

Since the implementation of the malaria elimination work in our city, the malaria prevention and control work has achieved remarkable results, which has improved the people's knowledge of malaria and self-prevention ability, and greatly enhanced the medical institutions' ability to treat malaria cases. The indicators reached the standard of eliminating malaria. To maintain this goal, the following points need to be noted.

With socio-economic development, urban expansion has accelerated and regional ecological changes have resulted in a decrease in malaria cases and the elimination of indigenous cases, this point was confirmed in the study of Wang et al. Wang reported that malaria prevention and control measures and local fiscal revenue increases were related to the decrease of malaria incidence in Hainan [19].

In 2017, China reported zero indigenous malaria case for the first time. In addition, zero indigenous malaria case was reported in the Chinese mainland for three consecutive years from 2017 to 2019 [9]. However, malaria cases imported from 
Africa and Southeast Asia are still occurring in China because of overseas labor [20, 21]. Therefore, country-led efforts are regional and intersectoral cooperation and ongoing monitoring and evaluation. Meanwhile, some works must be done consistently, such as the maintenance of non-transmission status, the diagnosis, treatment and management of imported malaria cases, the implementation of business training, technical guidance, quality control and supervision and inspection, through multi-sectoral collaboration [22, 23].

Maintaining the microscopic capability of primary health inspectors is also a challenge in achieving and sustaining malaria eradication [24]. In 2011, the completion rate of blood tests was $81.7 \%$ (13391/16387), and there were nine unclassified plasmodium infections during 2007-2018. Hence, the skills of microscopists for the preparation and interpretation of blood smears should be strengthened. In order to keep the eradication of malaria sustainable, we will take precise measures at different levels and areas. The Internet and other technological means should be used to enhance the level of information technology and improve the effectiveness of the prevention[25].

\section{Conclusions}

Our study found that the source of malaria case detection has changed greatly from the control to elimination stage, and the individual case-based malaria surveillance system generally worked well in the malaria elimination stage in North China. In the long term, systematic vector surveillance to control adult mosquito density to less harmful levels is also an important part of the post-elimination phase of malaria. However, The North China still faces many challenges, including epidemiological changes in malaria cases among the international migrant workers and hard-to-reach 
populations.

\section{Acknowledgements}

354 This study was supported by the Doctoral Research Foundation of Hebei University of Engineering, Hebei Provincial Centre for Disease Control and Prevention, Handan

Municipal Science \& Technology Commission.

\section{Authors' contributions}

RG, ZJ conducted the study design and study implementation. RG, SL, ZJ performed the data analyses and draft the manuscript. RG, JZ, XW, JL, WZ, SL, ZJ provided public health insight for analyzing the results. All authors read, edited and approved the final manuscript.

\section{Funding}

This study was supported by the Special Research Foundation of Hebei University of

365 (19422083007, 20312904044).

\section{Data availability}

367 Not applicable.

\section{Ethical approval}

369 Not required.

\section{Consent for publication}

371 Not applicable.

\section{Competing interests}

373 The auothers declare that they have no competing interests. 


\section{References}

[1] Zofou, D., Nyasa, R. B., Nsagha, D. S., Ntie-Kang, F., et al., Control of malaria and other vector-borne protozoan diseases in the tropics: enduring challenges despite considerable progress and achievements. Infect Dis Poverty 2014, 3, 1-1.

[2] Lai, S., Sun, J., Ruktanonchai, N. W., Zhou, S., et al., Changing epidemiology and challenges of malaria in China towards elimination. Malar J 2019, 18, 107-107.

[3] Rossati, A., Bargiacchi, O., Kroumova, V., Zaramella, M., et al., Climate, environment and transmission of malaria. Le infezioni in medicina 2016, 24, 93-104.

[4] Cotter, C., Sturrock, H. J., Hsiang, M. S., Liu, J., et al., The changing epidemiology of malaria elimination: new strategies for new challenges. Lancet 2013, 382, 900-911.

[5] Lu, G., Zhou, S., Horstick, O., Wang, X., et al., Malaria outbreaks in China (1990-2013): a systematic review. Malar J 2014, 13, 269-269.

[6] Ndong, I. C., Okyere, D., Enos, J. Y., Amambua-Ngwa, A., et al., Challenges and perceptions of implementing mass testing, treatment and tracking in malaria control: a qualitative study in Pakro sub-district of Ghana. BMC Public Health 2019, 19, 695-695.

[7] Zhou, Z. J., The malaria situation in the People's Republic of China. Bull World Health Organ 1981, 59, 931-936.

394 [8] Yin, J.-H., Zhou, S.-S., Xia, Z.-G., Wang, R.-B., et al., Chapter One - Historical Patterns of Parasitology, Academic Press 2014, pp. 1-19.

[9] Feng, J., Zhang, L., Huang, F., Yin, J.-H., et al., Ready for malaria elimination: zero indigenous case reported in the People's Republic of China. Malar J 2018, 17, 315-315.

400 [10] Cao, J., Sturrock, H. J. W., Cotter, C., Zhou, S., et al., Communicating and monitoring surveillance and response activities for malaria elimination: China's "1-3-7" strategy. PLoS medicine 2014, 11, e1001642-e1001642.

[11] Feng, X.-Y., Xia, Z.-G., Vong, S., Yang, W.-Z., Zhou, S.-S., Chapter Four - Surveillance and Response to Drive the National Malaria Elimination Program, in: Zhou, X. N., Kramer, R., Yang, W. Z. (Eds.), Advances in Parasitology, Academic Press 2014, pp. 81-108. and response. Infect Dis Poverty 2013, 2, 1-1.

[13] Malone, J. B., Bergquist, R., Martins, M., Luvall, J. C., Use of Geospatial Surveillance and 4,15 . 
[14] Vatandoost, H., Raeisi, A., Saghafipour, A., Nikpour, F., Nejati, J., Malaria situation in Iran: 2002-2017. Malar J 2019, 18, 200-200.

[15] Huang, Q., Hu, L., Liao, Q.-B., Xia, J., et al., Spatiotemporal Analysis of the Malaria Epidemic in Mainland China, 2004-2014. Am J Trop Med Hyg 2017, 97, 504-513.

[16] Tatem, A. J., Jia, P., Ordanovich, D., Falkner, M., et al., The geography of imported malaria to non-endemic countries: a meta-analysis of nationally reported statistics. Lancet Infect Dis 2017, $17,98-107$.

[17] Herrador, Z., Fernandez-Martinez, B., Quesada-Cubo, V., Diaz-Garcia, O., et al., Imported cases of malaria in Spain: observational study using nationally reported statistics and surveillance data, 2002-2015. Malar J 2019, 18, 230.

[18] Zhang, S.-S., Zhou, S.-S., Zhou, Z.-B., Chen, T.-M., et al., Monitoring of malaria vectors at the China-Myanmar border while approaching malaria elimination. Parasit Vectors 2018, 11, 511-511.

[19] Wang, S.-Q., Li, Y.-C., Zhang, Z.-M., Wang, G.-Z., et al., Prevention measures and socio-economic development result in a decrease in malaria in Hainan, China. Malar J 2014, 13, $362-362$.

[20] Kounnavong, S., Gopinath, D., Hongvanthong, B., Khamkong, C., Sichanthongthip, O., Malaria elimination in Lao PDR: the challenges associated with population mobility. Infect Dis Poverty 2017, 6, 81-81.

[21] Wang, D., Li, S., Cheng, Z., Xiao, N., et al., Transmission Risk from Imported Plasmodium vivax Malaria in the China-Myanmar Border Region. Emerg Infect Dis 2015, 21, 1861-1864.

[22] Zhang, X., Yao, L., Sun, J., Pan, J., et al., Malaria in Southeastern China from 2012 to 2016 : Analysis of Imported Cases. Am J Trop Med Hyg 2018, 98, 1107-1112.

[23] Zhou, S., Li, Z., Cotter, C., Zheng, C., et al., Trends of imported malaria in China 2010-2014: analysis of surveillance data. Malar J 2016, 15, 39-39.

[24] Ding, G., Zhu, G., Cao, C., Miao, P., et al., The challenge of maintaining microscopist capacity at basic levels for malaria elimination in Jiangsu Province, China. BMC Public Health 2018, 18, 489-489.

[25] Sahu, M., Tediosi, F., Noor, A. M., Aponte, J. J., Fink, G., Health systems and global progress towards malaria elimination, 2000-2016. Malar J 2020, 19, 141-141. 